\title{
Tsafon
}

Revue d'études juives du Nord

$81 \mid 2021$

Des synagogues à travers les âges Lieux de prières, lieux d'études et autres fonctions

\section{La synagogue médiévale de Montpellier}

\section{Michaël lancu}

\section{(2) OpenEdition \\ Journals}

Édition électronique

URL : https://journals.openedition.org/tsafon/3689

DOI : 10.4000/tsafon.3689

ISSN : 2609-6420

Éditeur

Association Jean-Marie Delmaire

Édition imprimée

Date de publication : 1 juillet 2021

Pagination : 39-48

ISSN : 1149-6630

\section{Référence électronique}

Michaël lancu, «La synagogue médiévale de Montpellier », Tsafon [En ligne], 81 | 2021, mis en ligne le 01 juillet 2021, consulté le 15 septembre 2021. URL : http://journals.openedition.org/tsafon/3689 ; DOI : https://doi.org/10.4000/tsafon.3689 


\title{
La synagogue médiévale de Montpellier
}

\author{
Michaël Iancu*
}

\section{La présence juive médiévale à Montpellier}

La présence juive à Montpellier remonte sans doute à la création de la ville et l'habitat juif y est concentré en deux quartiers distincts : dans la partie royale qui se trouve près du palais des Guilhem et dans l'espace seigneurial ecclésiastique qui est localisé vers Montpelliéret.

La documentation sur les juifs résidant dans la partie royale de la ville est assez importante grâce à divers travaux sur leur histoire et à la publication d'archives. À commencer par celle, en 1889, de Salomon $\mathrm{Kahn}^{1}$, rabbin de Nîmes, concernant le premier document, en latin, qui relate l'existence de la sinagoga judeorum de la rue Sabatarié-Neuve à la fin du XIII ${ }^{\mathrm{e}}$ siècle (1277), puis vinrent les études de Louise Guiraud ${ }^{2}$ qui fit connaître, en 1899, des plans détaillés des installations successives des juifs dans le temps et dans l'espace - plans à revoir toutefois. Ces travaux pionniers furent suivis d'autres recherches qui tentèrent de cerner l'habitat

\footnotetext{
* Docteur en histoire, directeur de l'Institut Universitaire Maïmonide, Averroès, Thomas d'Aquin, Montpellier.

Cet article reprend en partie ma contribution à l'ouvrage Présence juive en Bas-languedoc médiéval. Dictionnaire de géographie historique, par Michaël Iancu et Danièle IancuAgou, avec la collaboration de Pierre-Joan Bernard, Préface de Daniel Le Blévec, Cartographie d'Ephrem Hurel, Paris, Cerf-Patrimoines, Collection Nouvelle Gallia Judaica $\mathrm{n}^{\circ} 12$, à paraître fin 2021.

${ }^{1}$ Salomon Kahn, « Documents inédits sur les juifs de Montpellier au Moyen Âge », Revue des études juives, tome 19,1889 , p. 270 . Cf. également les numéros suivants : tome 22 , 1891 , p. 264-279; tome 23, 1891, p. 265-278; tome 28, 1894, p. 118-141.

${ }^{2}$ Louise Guiraud, "Recherches topographiques sur Montpellier au Moyen Âge », dans Mémoires de la Société archéologique de Montpellier, $2^{\mathrm{e}}$ série, t. 1 ; 1899.
} 
des juifs montpelliérains avant leur éviction (1306) et après, période ponctuée de retours ${ }^{3}$. Actuellement encore, historiens et archéologues continuent de sonder le passé des «quartiers juifs» montpelliérains au Moyen Âge ${ }^{4}$.

Mais auparavant, d'autres érudits ont conservé les traces d'un ensemble cultuel hébraïque médiéval, à Montpellier. Dès le XVII ${ }^{\mathrm{e}}$ siècle, dans une monographie sur la ville, le chanoine Pierre Gariel en remarque la présence ${ }^{5}$. Puis c'est au tour de Charles d'Aigrefeuille, en 1737, de décrire précisément une composante de cet ensemble ${ }^{6}$, le mikvé, ou bain rituel, soit une description fort tardive, près de quatre siècles après sa mise en service. Repose-t-elle sur la tradition orale d'une durée exceptionnelle ou l'auteur aurait-il eu accès à des documents aujourd'hui perdus ? En voici un extrait :

Le plus ancien monument qu'ils nous ayent laissé, se voit dans la maison de Montade, qui se présente en face, lorsqu'on vient de la rue du Puits des Esquilles. On y trouve des voûtes souterraines, qui répondent à un grand puits, d'où l'on tiroit de l'eau pour servir à la purification des femmes juives ; tout alentour, elles avoient des cabinets pour se déshabiller; \& dans les murailles de ces cabinets, il y a des niches, où l'on mettoit du feu pour les chauffer, \& des lampes pour les éclairer. À côté, on trouve une plus grande voûte, où il y a quatre ouvertures au haut, par où les femmes juives entendoient la prédication du Rabin, de la même manière qu'elles font encore dans la juiverie d'Avignon.

Le récit de Charles d'Aigrefeuille est repris par des historiens plus récents qui témoignent des transformations subies par ce mikvé. Léon Coste rapporte que le locataire avait fait murer la fenêtre géminée pour se protéger de l'humidité qu'elle lui occasionnait ${ }^{7}$. Quant à Louis Escuret, il

\footnotetext{
${ }^{3}$ Il s'agit de travaux émanant d'érudits locaux comme Léon Coste, Les transformations de Montpellier depuis la fin du XVII siècle, Montpellier, 1893 ; Émile Aezmard, Étude sur les Israélites de Montpellier au Moyen Âge, Nîmes, 1924 ; Louis Escuret, Vieilles rues de Montpellier, Montpellier, 1964.

${ }^{4}$ Voir la bibliographie en fin d'article.

${ }_{5}^{5}$ Pierre Gariel, Idée générale de la Ville de Montpellier recherchée et présentée aux honestes gens, Montpellier, 1665, p. 4-5.

${ }^{6}$ Charles d'Aigrefeuille, Histoire de la ville de Montpellier depuis son origine jusqu'à nos temps, Montpellier, Jean Martel, 1737, 2 vol.

On possède également une description des bassins de purification de la synagogue d'Avignon grâce à Thomas Platter qui les visita en 1598. Cf. Félix et Thomas Platter à Montpellier 1552-1559, 1595-1599. Notes de voyage de deux étudiants bâlois publiées d'après des manuscrits originaux appartenant à la Bibliothèque de l'Université de Bâle, Montpellier, 1892.

${ }^{7}$ Léon Coste, Les transformations de Montpellier, op. cit., qui visita les lieux en 1887.
} 
fait état, vers 1960, d'un endroit dénaturé par les gravats et l'amoncellement de détritus de toutes sortes ${ }^{8}$.

Restauré en 1985 à l'occasion du millénaire de la ville de Montpellier et grâce à l'action de Georges Frêche, député-maire, puis ouvert au public ${ }^{9}$, ce bain rituel est désormais éclairé par une mention latine retrouvée récemment par Jean-Louis Vayssettes ${ }^{10}$ dans un testament du 28 avril 1292 des archives municipales, sur les balneis judeorum « correspondant exactement au mikvé de la rue de la Barralerie ». Relevons « du bain juif » et non des « bains juifs », dénomination impropre ${ }^{11}$.

\section{La synagogue des juifs du roi}

Le 8 juillet 1277, un acte de vente est signé par les représentants de la communauté juive de Montpellier concernant une maison «sita in Montepessulano juxta synagoga judeorum », se trouvant sur les hauteurs de la ville dans son quartier commerçant, propriété de Jacques II d'Aragon. L'endroit dépendait de l'autorité des Guilhem (puis des rois d'Aragon et de Majorque). Les représentants de la communauté juive portaient des noms qui laissent supposer une origine des collectivités voisines comme Jacob de Lunel, Tauros de Beaucaire, Ferrier Bonafos, Bondia de Beaucaire, Isaac de Loderva et Abram de Béziers. Destiné à devenir une «maison d'aumône» (domus helemosine), cet édifice manifeste l'existence d'une synagogue dans la rue de la Barralerie - «barraliers » signifiant tonneliers - en fonction donc à la fin du XIII ${ }^{\mathrm{e}}$ siècle et très probablement auparavant.

Cette source est relativement avare de détails sur la situation topographique de la maison. En revanche, un autre document daté du 8 juillet 1301 précise que la synagogue se trouvait « in Montepessulano in loco vocato Castrum Mutonis », c'est-à-dire dans le quartier de CastelMoton. Ce sont les minutes du notaire qui, vers 1293-1301, révèlent des

\footnotetext{
${ }^{8}$ Louis Escuret, Vieilles rues de Montpellier, op.cit.

${ }^{9}$ À l'occasion du colloque organisé par Carol Iancu dont les actes ont été publiés : Les juifs à Montpellier et dans le Languedoc du Moyen Âge à nos jours, Montpellier, Université Paul Valéry, 1988.

10 Jean-Louis Vayssettes, «Une mention médiévale des bains juifs de Montpellier et nouvelles interprétations topographiques », dans Danièle Iancu-Agou (dir. avec la collaboration d'Élie Nicolas), Philippe le Bel et les juifs du royaume de France (1306), Paris, Cerf, collection Nouvelle Gallia Judaica n ${ }^{\circ}$, 2012, p. 31-41.

${ }^{11}$ Cf. l'article récent de Danièle Iancu-Agou, «L'historiographie contemporaine autour du mikvé médiéval de Montpellier », dans Jean Nougaret le Languedocien (1939-2013). Hommages à sa mémoire, Etudes Héraultaises, nº7, 2016, p. 96-99.
} 
actes de location, par des juifs, de demeures propriétés de chrétiens, rue du Castel-Moton prouvant l'extension de l'habitat juif vers la rue actuelle du Palais coupée par celle du Puits-des-Esquilles (le chanoine d'Aigrefeuille l'atteste en 1737) et par la ruelle qui la prolonge, le tout formant le carrefour de Castel-Moton ${ }^{12}$.

Dans une récente communication, Pierre-Joan Bernard a souligné l'importance de la mention d'une " école des juifs » à Castel-Moton dès 1201 ainsi que celle «d'un four près de la synagogue », pour CastelMoton, soit un siècle plus tard ${ }^{13}$. Peut-on en conclure que la synagogue attestée en 1277 (proche du mikvé de la Barralerie) et présente encore en 1302, pouvait s'ouvrir par la venelle sur la rue du Puy des Esquilles et vers le quartier de Castel-Moton? Les fouilles à venir devraient donc tenir compte de ces conclusions et s'orienter géographiquement vers le quartier de Castel-Moton.

Ainsi le croisement des sources tant hébraïques (citons Benjamin de Tudèle qui au cours de ses pérégrinations, au XII ${ }^{\mathrm{e}}$ siècle, constate la présence de "maisons d'études ») que latines (avec la révélation d'une synagogue, maison de charité, d'un mikvé fin XII' siècle, d'un four au début du XIV ${ }^{\mathrm{e}}$ ) permet d'affirmer que les juifs habitent au cœur de la ville, dans « l'Écusson », et y vivent leur âge d'or intellectuel. Cet habitat n'est nullement cloisonné, c'est un quartier pourvu de bâtiments cultuels et ouvert, à la différence de ce que seront les ghettos de l'époque de la Renaissance.

En résumé, grâce aux diverses mentions dans les sources archivistiques, nous pouvons établir la chronologie de la présence juive pour la seule partie royale, dans Montpellier. Pour le XIII ${ }^{\mathrm{e}}$ siècle, quatre mentions concernent l'îlot de Castel-Moton : 1201, "école des juifs »; 1277, «maison de l'Aumône » près de la «sinagoga judeorum » dans l'actuelle rue de la Barralerie; 1292, «balneis judeorum»; 1302, « sinagoga in loco voccato Castro Mutonis ", proche du « four ». Près d'un siècle plus tard, en 1387, lors de la dernière période durant laquelle

\footnotetext{
${ }^{12}$ Cf. S. Kahn, «Documents inédits... », Revue des études juives, 28, 1894, p. 123, et p. 125 et pièce justificative XIII, p. 137-141, et Danièle Iancu, « Le mikvé et l'évolution du quartier juif médiéval à Montpellier», dans Les Juifs à Montpellier et dans le Languedoc, op. cit., Carol Iancu (dir.), 1988, p. $72-92$ (ici p. 81).

13 «Le quartier seigneurial de Castel-Moton: aux origines de Montpellier et de la présence juive dans la ville », sujet traité lors d'un Lundi du LEM-Montpellier, le 5 mars 2018, à l'Institut Universitaire Maïmonide, Averroès, Thomas d'Aquin (IUMAT), en la salle Profiat, rue de la Barralerie, là où, en sous-sol, se trouve le mikvé remontant au XII siècle.
} 
la présence des juifs est autorisée, cette communauté vit toujours dans le même secteur, rue de la Vieille Intendance. Entre-temps, les juifs avaient été victimes de spoliations de leurs biens puis d'une brutale expulsion en 1306, édictée par Philippe le Bel ; entre 1315 et 1322 ainsi qu'entre 1359 et 1394 ils furent autorisés à revenir avant que Charles VI les chasse définitivement du royaume de France.

Une synagogue, qui comme son nom l'indique est le lieu de rassemblement de la communauté juive, renseigne-t-elle sur la dimension démographique de ladite communauté ? Pour les prières, un quorum de dix hommes (le myniane) est requis, est-ce suffisant pour définir quantitativement une communauté juive ? Et à partir de quel nombre, un groupe de juifs décidait-il d'édifier un établissement cultuel ? Sachant que toute maison peut abriter un oratoire qui suffit au culte. Il est donc évident qu'un édifice de l'ampleur de celui de Montpellier a pu être érigé grâce à une communauté importante, même si ces lieux ne se différenciaient pas extérieurement d'un édifice civil.

\section{Les fouilles récentes autour du mikvé pour retrouver la synagogue}

Depuis sa restauration et son ouverture au public en 1985, le site du mikvé a fait l'objet de trois campagnes de fouilles, en 2000 et en 2009, pour lesquelles un résumé a été présenté à un colloque en 2011 du Musée d'Art et d'Histoire du Judaïsme organisé et publié par Paul Salmona et Laurence Sigal ${ }^{14}$; et la dernière campagne s'est déroulée en $2016^{15}$. Il en ressort que, finalement, la localisation précise du lieu synagogal demeure difficile à déterminer malgré cinq citations dans des documents d'archives et alors que ceux-ci délimitent sa présence dans un même périmètre restreint, limitrophe à la Barralerie où se trouve le mikvé, seul point d'appui incontournable sur lequel doivent se fonder les recherches.

Il suffit de citer Christian Markiewicz, dans son rapport de 2017 sur la phase 3 des fouilles. Selon lui, la recherche archéologique sur le site de la synagogue de Montpellier est :

\footnotetext{
${ }^{14}$ Michaël Iancu, «La synagogue médiévale de Montpellier », dans Paul Salmona et Laurence Sigal (dir.), L'archéologie du judaïsme en France et en Europe, Paris, La Découverte, MAHJ et INRAP, 2011, p. 169-176.

${ }^{15}$ Christian Markiewicz, Heike Hansen, Étude archéologique de l'ensemble hébraïque médiéval, rue de la Barralerie, Étude complémentaire, phase 3, Ville de Montpellier (34), Mission Grand Cœur, DRAC-SRA/CRMH Région Occitanie, Phase 3, 2017, p. 1-122. Que soit ici remercié C. Markiewicz qui nous a communiqué le document final de synthèse.
} 
[...] emblématique de la difficulté de retrouver les traces matérielles d'une histoire marquée par les coupures brutales et douloureuses que connut la vie des communautés juives au cours des derniers siècles du Moyen Âge. À l'issue des différentes études conduites en sous-sol (sondages), ou dans les différentes élévations, il apparaît assez clairement que le site a subi de profondes transformations (dès les phases les plus anciennes d'occupation révélées et qui remontent au XIII ${ }^{e}$ siècle). Trois grandes périodes d'occupation du site sont mises en évidence : la période d'utilisation du mikvé en tant que bain rituel, puis l'utilisation de la grande salle médiévale du bâtiment nord qui marque un état plus tardif correspondant à une restructuration, enfin, la période du XVIII . siècle.

Curieusement, un autre bassin de 3,40 m de longueur, associé au mikvé, suscite d'autres interrogations. Aurait-il eu d'autres fonctions que celle d'un bain rituel, comme la cashérisation de la vaisselle ? En d'autres lieux des bassins ont eu diverses destinations comme à Carpentras où un bain était réservé à la toilette ou encore à Syracuse où des bains rupestres étaient associés aux cuves rituelles. C. Markiewicz laisse espérer des conclusions prometteuses :

Nul doute [poursuit-il] qu'accompagné d'un programme de restauration adapté, cet ensemble offrira à l'avenir un potentiel des plus intéressants permettant de faire le lien entre la période de présence de la communauté juive et l'étape historique qui lui fit suite. Bien représentée à Montpellier, cette problématique liée à la mutation des quartiers juifs au XIV ${ }^{\mathrm{e}}$ siècle ne trouve pas de comparaison ailleurs, au sein des études conduites dans ce registre innovant de la recherche, et s'impose par conséquent comme l'un des exemples phares.

Les fouilles successives conduites à Montpellier confirment donc que la localisation d'une synagogue médiévale est ardue à établir avec précision ${ }^{16}$. Comment retrouver un tel monument et surtout comment comprendre son articulation ? Ceci d'autant plus que les sources notariales ou judiciaires ne renseignent pas sur l'architecture, voire les matériaux des biens juifs synagogaux ou privés qui permettraient de mieux identifier les vestiges.

En outre, à Montpellier comme ailleurs, après le départ des juifs, divers aménagements ont, au fil des temps, modifié, voire dénaturé, l'ordonnance initiale des lieux et leur vocation originelle. Toutefois, les recherches archéologiques mettent en évidence des éléments qui prouvent

\footnotetext{
${ }^{16}$ Claude de Mecquenem, «Les synagogues médiévales françaises : à la recherche d'un patrimoine architectural disparu ?», Religions et Histoire, n ${ }^{\circ} 12$, 2007, éd. Faton, p. 2023.
} 
que le lieu de culte juif est proche des endroits fouillés. Sans doute faudrait-il davantage s'orienter vers l'îlot de Castel-Moton, puisque le bâtiment historique est orienté vers la venelle y conduisant, avec l'imposant portail d'entrée - dont l'origine médiévale n'est que supposée - de la grande pièce du rez-de-chaussée appelée « salle des trois Arches ».

Il reste donc à faire preuve d'une grande prudence envers des vestiges connus grâce une tradition locale qui ne s'accorde pas toujours avec la connaissance scientifique des lieux. Les données d'archives doivent faire face à une mémoire orale, collective et souvent floue proche de la légende ${ }^{17}$.

Le mikvé du XII ${ }^{\mathrm{e}}$ siècle de Montpellier peut actuellement être considéré comme le joyau touristique de la cité. Il reste le site le plus visité de la «Ville du Mont », immédiatement après la Crypte Notre-Dame des Tables, contemporaine du bain rituel ${ }^{18}$. La municipalité n'abandonne pas le projet d'un site muséal ou d'un parcours muséal dans le quartier juif de la Barralerie où, en 2008, des vitrines didactiques destinées à sensibiliser les Montpelliérains et les passants avaient été mises en place. Une telle conscience municipale du passé juif se traduit-elle aujourd'hui dans les livres d'histoire ${ }^{19}$ ? Il semble que oui : alors que l'historien Gérard Cholvy, dans son Histoire de Montpellier de 1984, ne consacrait que quelques lignes à la présence juive, Christian Amalvi et Rémy Pech, directeurs de la nouvelle Histoire de Montpellier parue en 2015, accordent généreusement trois chapitres ${ }^{20}$ au passé juif montpelliérain et s'attardent

17 Danièle Iancu-Agou, «Le patrimoine juif médiéval en France: entre histoire, archéologie et tradition orale », dans Paul Salmona et Laurence Sigal (dir.), L'archéologie du judaïsme en France et en Europe..., op. cit., p. 81-91; et «Entre documents et monuments, mémoire orale, mémoire vécue : réflexions autour de la Provincia judaica », dans Le plaisir de l'art du Moyen Âge. Mélanges en hommage à Xavier Barral i Altet, Paris, éd. Picard, 2012, p. 946-951. Cf. aussi de Noël Coulet, sa préface à la Provincia judaica, Dictionnaire de géographie historique des juifs en Provence médiévale, ParisLouvain, Peeters, 2010.

${ }^{18}$ Mentionnée pour la première fois en 1090, la dénomination même de « Notre Dame des Tables » apparaît officiellement le 15 août 1204, lors de la fête de Marie. Dans cette église, Guillaume Grimoard, le futur Urbain V, y recevra, à la Toussaint 1342, les insignes de son doctorat en décrets (droit canon).

${ }^{19}$ Laurence Sigal et Paul Salmona, «Les juifs, effacés de l'Histoire ? », Le Monde des Religions, janvier 2017. Idem, Le judaïsme : une tache aveugle dans le récit national, ouvrage issu du colloque organisé les 17 et 18 janvier, Paris, Albin Michel et MAHJ, mars 2021.

${ }^{20}$ Michaël Iancu, « Le judaïsme à Montpellier au Moyen Âge », « La communauté juive à l'époque moderne » et «Les épreuves de la guerre et la renaissance du judaïsme à Montpellier », dans Christian Amalvi et Rémy Pech (dir.), Histoire de Montpellier, 
sur la fameuse polémique de 1230 survenue inter judeos autour du Guide de Maïmonide, dont Hillel de Vérone (XIII ${ }^{\mathrm{e}}$ siècle) ${ }^{21}$ a rapporté la destruction en place publique. Par ailleurs, les publications du Midi Libre viennent de sortir un hors-série édition 2020/2021 intitulé Juifs d'Occitanie. Une histoire millénaire ${ }^{22}$, destiné à être largement diffusé dans les kiosques et les établissements scolaires.

\section{Compléments bibliographiques}

- Ayasse A., Gomez M., Hartmann-Virnich A., Markiewicz Ch., PribetichAznar Cl., Étude archéologique du complexe médiéval, 1, rue Barralerie, Phase I. rapport final d'opération, Aix-en-Provence, LAMM, CNRS UMR 6572, mai 2010.

- Gensac Alain, «L'ensemble cultuel hébraïque médiéval de Montpellier (France) », dans Max Polonovski (éd.), Le Patrimoine juif européen, Actes du Colloque international tenu à Paris (MAHJ, avril 1999), ParisLouvain, éd. Peeters, 2002, p. 285-305.

- Huser Astrid, avec la collaboration de Jean-Louis Vayssettes et de Danièle Iancu-Agou, « Document final de synthèse. Étude du bâti », Mikvé et synagogue : quartier médiéval juif à Montpellier (Hérault), Montpellier, ministère de la Culture et de la Communication, Service régional de l'archéologie/AFAN Méditerranée-Nîmes/ Ville de Montpellier, 2000.

- Iancu-Agou Danièle, Provincia judaica. Dictionnaire de géographie historique des juifs en Provence médiévale, Préface de Noël Coulet, Paris-Louvain, Peeters, 2010.

Toulouse, Privat, 2016, respectivement p. 97-107, 211-212, et 575-580. Cf. l'argumentation de Danièle Iancu-Agou dans «Topographie historique des juiveries médiévales : l'entreprise des dictionnaires régionaux », Les juifs. Une tache aveugle... op.cit., p. 184-193 (ici p. 191-193).

${ }^{21} \mathrm{Cf}$. nos résumés des controverses, Michaël Iancu, Les juifs de Montpellier et des terres d'Oc, Figures médiévales, modernes et contemporaines, Paris, Cerf, 2014, p. 31 et suivantes ; et dans Les juifs des terres d'Oc, Collection Les études du Crif, janvier 2020, $\mathrm{n}^{\circ} 57$, p. 22-23.

22 Juifs d'Occitanie. Une histoire méconnue, «Hors-Série» du Midi Libre, édition 2020/2021, 115 pages. 
- Iancu-Agou Danièle, « Georges Duby (1919-1996) et le renouveau des études sur les juifs de Provence », Revue des études juives, 159 (3-4), 2000, p. 479-483.

- Markiewicz Christian, Hansen Heike, Étude archéologique de l'ensemble hébraïque médiéval, rue de la Barralerie, Étude complémentaire, phase 3, Ville de Montpellier (34), Mission Grand Cœur, DRAC-SRA/CRMH Région Occitanie, Phase 3, 2017, p. 1-122.

- Schwarzfuchs Simon, «Coup d'œil sur la littérature rabbinique médiévale de Provence », dans Danièle Iancu-Agou, Élie Nicolas (éd.) Des Tibbonides à Maïmonide. Rayonnement des juifs andalous en pays d'Oc médiéval, Paris, Cerf, coll. Nouvelle Gallia Judaica nº 4, p. 83-91.

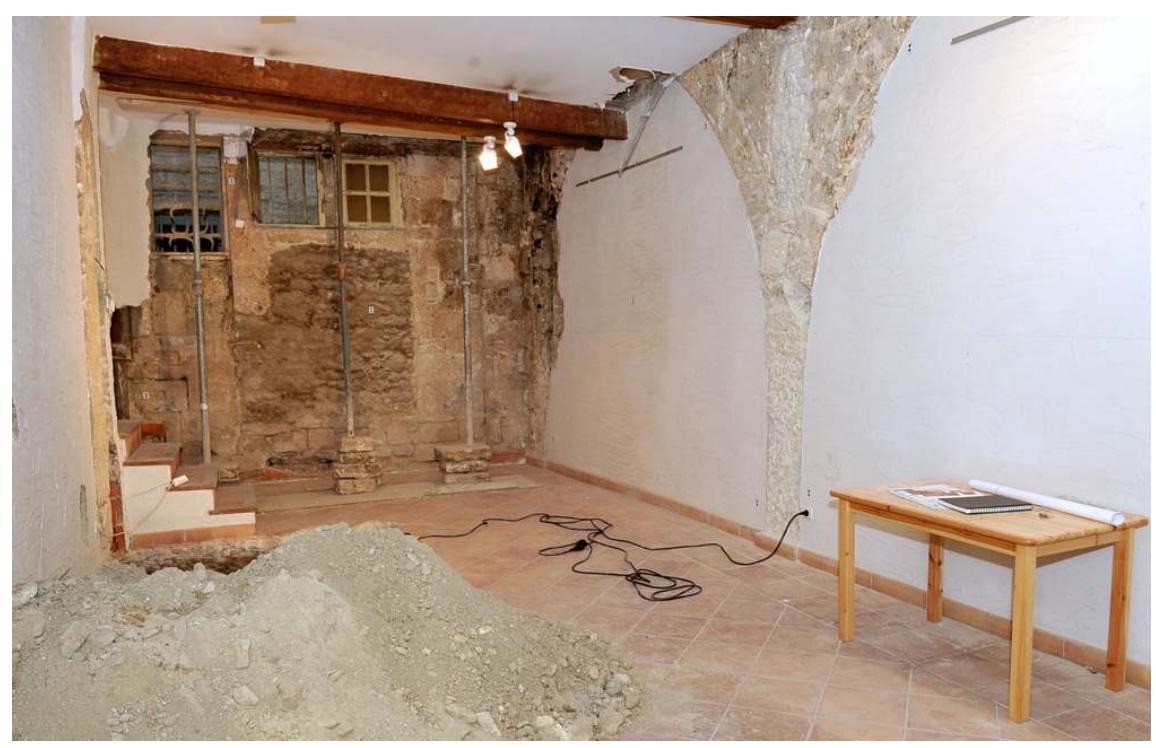

La «salle des trois Arches» lors des fouilles. Collection de l'Institut Universitaire Maïmonide, Averroès, Thomas d'Aquin, Montpellier. 


\section{Michaël Iancu}

Le mikvé de Montpellier

Collection de l'Institut Universitaire Maïmonide, Averroès, Thomas d'Aquin, Montpellier.

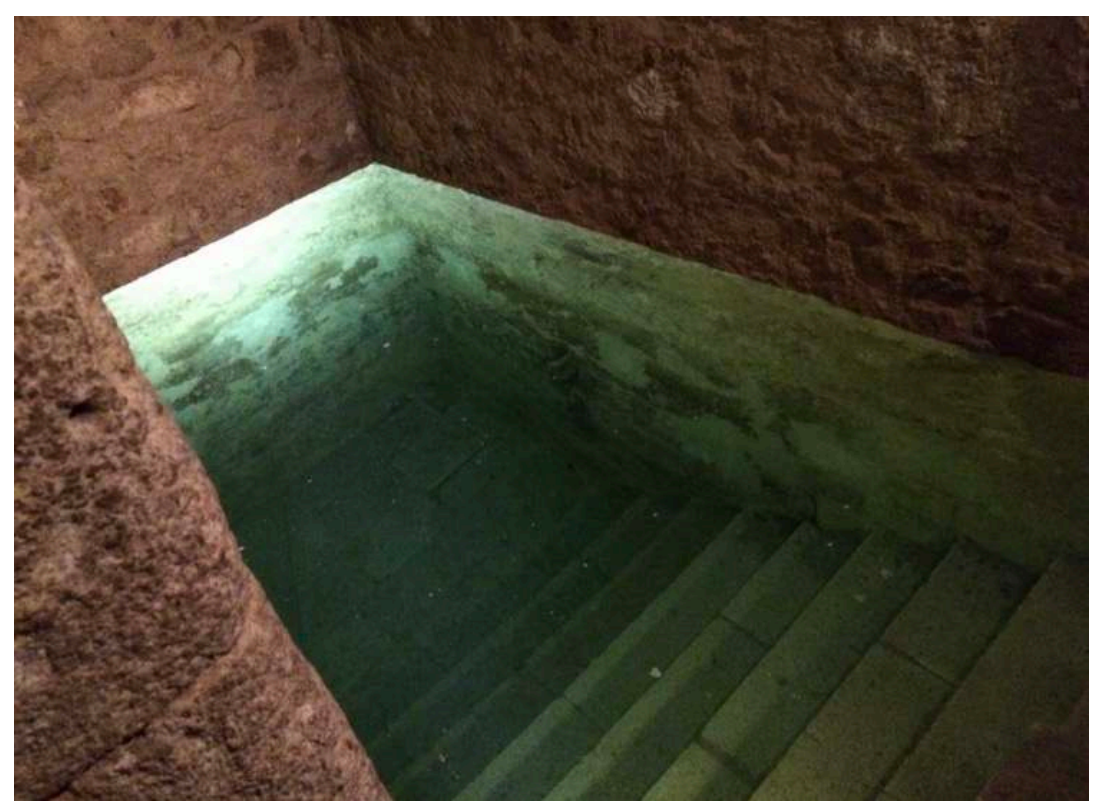

Le mikvé, alimenté par une nappe d'eau souterraine.

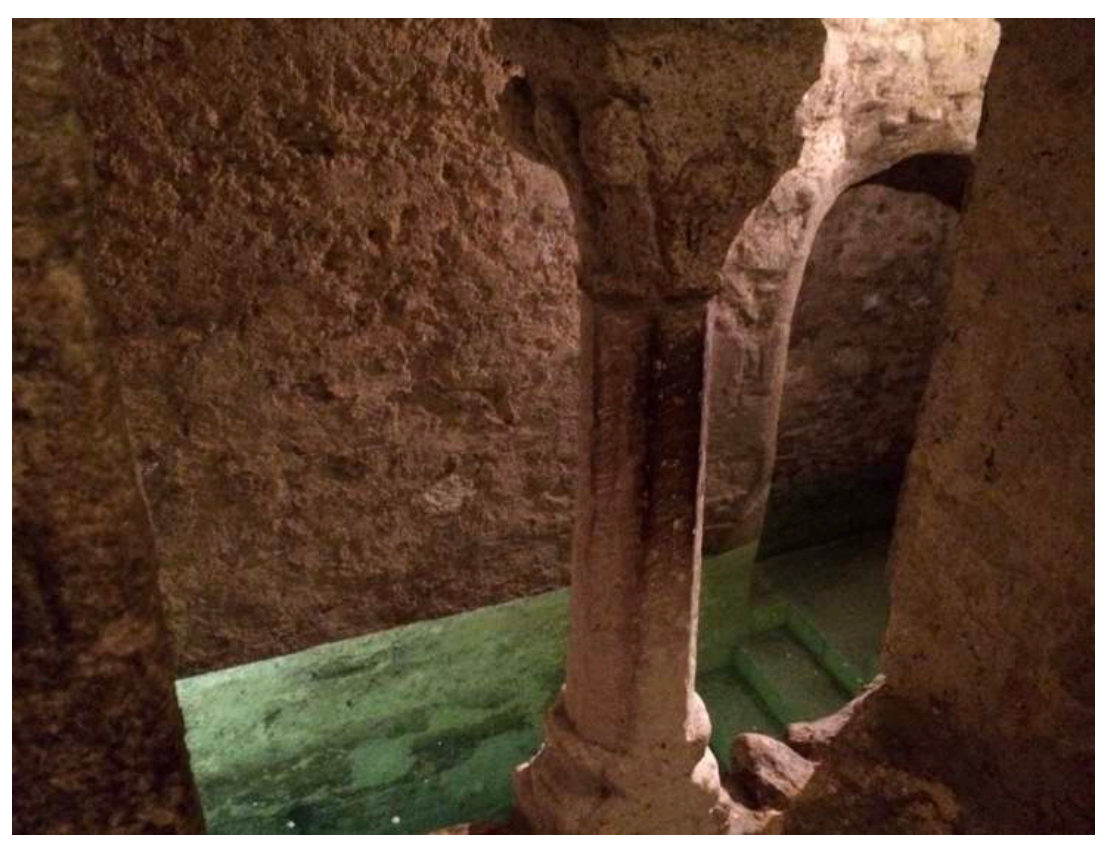

Arche de style roman bordant le mikvé. 